\title{
Nicotine-Haloperidol Interactions and Cognitive Performance in Schizophrenics
}

\author{
Edward D. Levin, Ph.D., William Wilson, Ph.D., Jed E. Rose, Ph.D., and Joseph McEvoy, M.D.
}

Nearly $90 \%$ of schizophrenics smoke cigarettes, considerably higher than the general population's rate of $25 \%$. There is some indication that schizophrenics may smoke as a form of self-medication. Nicotine has a variety of pharmacologic effects that may both counteract some of the cognitive deficits of schizophrenia and counteract some of the adverse side effects of antipsychotic drugs. In the current study, we assessed the interactions of haloperidol and nicotine on cognitive performance of a group of schizophrenics. These patients were in a double-blind study, randomly assigning them to low, moderate, and high dose levels of haloperidol. The subjects, all smokers, came to the laboratory on four different mornings after overnight deprivation from cigarettes. In a double-blind fashion, they were administered placebo, low ( $7 \mathrm{mg} /$ day), medium (14 $\mathrm{mg} /$ day), or high (21 $\mathrm{mg} /$ day) dose nicotine skin patches. Three hours after administration of the skin patch, the

KEY WORDS: Nicotine; Haloperidol; Memory; Reaction time; Schizophrenia

The very high rates of cigarette smoking among schizophrenics, nearly $90 \%$, has been noted in a variety of studies (Hughes et al. 1986; O'Farrell et al. 1983). Over the past 30 years, smoking in the general population in the United States has declined to about 25\% (Fiore 1992), but the smoking rates in certain groups including

From the Department of Psychiatry (EDL, WW, JER, JM) and Department of Pharmacology (EDL), Duke University Medical Center, Department of Research Service, VA Medical Center (JER), Durham, North Carolina.

Address correspondence to: Dr. Edward D. Levin, Neurobehavioral Research Laboratory, Department of Psychiatry, Box \#3412, Duke University Medical Center, Durham NC 27710.

Received August 21, 1995; revised September 28, 1995; accepted January $5,1996$. subjects were given a computerized cognitive test battery including: simple reaction time, complex reaction time (spatial rotation), delayed matching to sample, the Sternberg memory test, and the Conners continuous performance test (CPT). With the placebo nicotine patch, there was a haloperidol dose-related impairment in delayed matching to sample choice accuracy and an increase in response time on the complex reaction time task. Nicotine caused a dose-related reversal of the haloperidol-induced impairments in memory performance and complex reaction time. In the CPT, nicotine reduced the variability in response that is associated with attentional deficit. These results demonstrate the effects of nicotine in reversing some of the adverse side effects of haloperidol and improving cognitive performance in schizophrenia. (c) 1996 American College of Neuropsychopharmacology [Neuropsychopharmacology 15:429-436, 1996]

schizophrenics remains high. Nicotine, the principal psychoactive ingredient in tobacco, has a wide variety of pharmacologic effects. Some of these effects such as cognitive enhancement can be beneficial, despite the other adverse health consequences of tobacco use. Some smokers might use tobacco as a form of self-medication. It seems most likely that groups who smoke most heavily may be most likely to be engaging in self-medication. Several lines of evidence point to the likelihood for nicotine self-medication in schizophrenics (Lohr and Flynn 1992). The current study was conducted to determine the pharmacologic effects of nicotine on cognitive function in schizophrenics, whether it might reverse some of the cognitive deficits associated with schizophrenia and as well as deficits that result from antipsychotic medication.

Nicotine has been widely shown to be very effective in stimulating dopamine (DA) release (Andersson et al. 
1981a,b; Clarke 1990, Grenhoff et al. 1986; Lichtensteiger et al. 1982; Wonnacott et al. 1989). This may be an important effect with regard to schizophrenics given the disturbances of DA systems with the disease and given that classic antipsychotic drugs are potent DA receptor blockers. Recent evidence from our laboratory and others point to the relationship between cigarette smoking and DA systems. We have found that haloperidol causes a dose-related increase in ad lib smoking (McEvoy et al. 1995a). Others have found that in nonschizophrenic smokers haloperidol administration also increases smoking (Dawe et al. 1995; Jarvik et al. 1996). Interestingly, in another study we found that the atypical antipsychotic drug clozapine causes a significant decrease in ad lib smoking (McEvoy et al. 1995b). In the current study, we assessed the effects of nicotine administered via skin patches on cognitive performance in schizophrenics randomly assigned to three different dose levels of haloperidol to determine the effects of nicotine on cognitive function in schizophrenics and the interactive effect of nicotine with haloperidol.

Many studies have shown that nicotine improves cognitive performance in humans and experimental animals (Levin 1992). However, some studies have either not found improvements or have found nicotine-induced deficits (Levin 1992). The nature of the behavioral tasks, the regimen of nicotine administration, and the particular subject population may critically influence the result.

Cognitive deficits have been found to be associated with schizophrenia. These include problems with attention (Benedict et al. 1994; Pandurangi et al. 1994) and memory (Gras-Vincendon et al. 1994). Antipsychotic drugs have also been shown to impair memory processes (Cleghorn et al. 1990). Nicotine may reverse some of the cognitive deficits associated with schizophrenia as suggested by the interesting work by Adler et al. (1993). Additionally, because nicotine promotes dopamine release (Wonnacott et al., 1989), it may help reverse the cognitive deficits associated with dopamine blockade by antipsychotic drugs. The current study was designed to assess the interaction of nicotine with haloperidol with regard to cognitive function in schizophrenics. Drug effects on sensorimotor function, cognitive processing speed, spatial memory, verbal memory, and attentiveness were assessed.

\section{METHODS}

\section{Subjects}

At John Umstead State Hospital in Butner, North Carolina, there is an ongoing study to assess the dose-effect function of haloperidol. There were seven AfricanAmericans and eight Caucasians: three women and 12 men. Their average age was 38.9 years (range 20 to 58).
All of the subjects were smokers. They smoked an average of 1.3 packs per day when allowed to smoke freely and had been smoking an average of 21.8 (range 2 to 40 ) years. Their scores on the Fagerström test for nicotine dependence averaged 6.6 (range 5 to 8 ) They had an average of 4.5 (range 0 to 16) hospitalizations with an average of 10.4 (range 0 to 27) years since first hospitalization All patients had been admitted in an exacerbation of psychosis associated with noncompliance with prescribed antipsychotic medications. They remained free of antipsychotic medication for an additional 3 to 7 days in the hospital before haloperidol was started. During this 3- to 7-day baseline period, assessments of muscle tone were made on repeated occasions. This study was approved by the Duke University Internal Review Board, and all proper consent forms were obtained.

\section{Haloperidol Administration}

Haloperidol was started at a dose of $2 \mathrm{mg} /$ day. At 2- to 3-day intervals, the haloperidol dose was incremented by $2 \mathrm{mg} /$ day, until a clear increase in bradykinesia rigidity was noted on daily assessments of muscle tone. This was the neuroleptic threshoid (NT) dose. Because steady state blood levels of haloperidol were unlikely to be obtained with a schedule of dose incrementation every 2 to 3 days, we may have slightly overshot the actual neuroleptic threshold dose in some patients. The patients were randomly assigned to low (one-third of the NT dose), medium (the NT dose), and high (three times the NT dose) doses of haloperidol in a doubleblind fashion for 4 weeks. The average doses of haloperidol used were the low dose group $(n=6) 1.59 \pm$ $1.00 \mathrm{mg} /$ day (mean $\pm \mathrm{SD}$ ), the medium dose group $(n=$ 6) $4.67 \pm 2.42$, and high dose group $(n=3) 10.00 \pm 3.46$. The patients were on this level of haloperidol for approximately two weeks before the current study. Consistent with standard clinical practice, benztropine mesylate (Cogentin) was given when Parkinsonian-like side effects of haloperidol were a problem. The average doses of benztropine (used were for the low dose group $1.00 \pm 0.82 \mathrm{mg} /$ day, the medium dose group $1.67 \pm$ 0.82 , and high dose group $2.00 \pm 0.00$. There was not a significant correlation between haloperidol and benztropine dose $\left(r^{2}=0.06\right)$ and the main effect of haloperidol dose category on benztropine dose was not significant $(p=.14)$, but the linear trend of benztropine dose over haloperidol dose categories showed a nearly significant rise $(p<.08)$. No significant effects of benztropine dose were seen in any of the behavioral measures.

\section{Nicotine Administration}

The subjects were brought to the laboratory in the morning before they had smoked any cigarettes. They 
were administered a nicotine skin patch $\left(\right.$ Nicoderm $\left.{ }^{\circledR}\right)$ in a double-blind fashion. Patches delivering $0,7,14$, and $21 \mathrm{mg} /$ day were administered on consecutive mornings in a randomized counterbalanced fashion.

\section{Cognitive Tests}

Four tests from the Automated Neuropsychological Assessment Metrics (ANAM) battery (Reeves et al. 1993a, b) were given: simple reaction time, complex reaction time (spatial rotation), delayed matching to sample, and a modified Sternberg memory test. In the simple reaction time test, the subjects were asked to press a key as soon as possible after the appearance of a snowflake design on the computer monitor. In the complex reaction time test, the subjects were called upon to determine as quickly as possible whether a four-bar histogram pattern is the same as another pattern rotated by 90 or 270 degrees. In the delayed matching to sample test, the subjects were shown a $4 \times 4$ matrix of light and dark squares. Then, they were shown two patterns and were asked to identify the one they had just seen. In the Sternberg memory test, a set of two to six letters is shown in the middle of the screen, then single letters are displayed and the subject has to indicate whether they were present in the previously shown sample set.

After the ANAM battery, the subjects were given the Conners' continuous performance test (Conners 1995). This test was originally developed to assess attention deficit disorder (Conners 1994). The test takes approximately 14 minutes and calls on the subject to respond as quickly as possible to the appearance of letters on the monitor except for " $X$," for which they are to withhold responding. Critical measures are accuracy, response speed, and variability in response speed across testing blocks of the session and over different interstimulus intervals (1, 2, and 4 seconds).

\section{Data Analysis}

The response accuracy and response latency data were assessed by analysis of variance for repeated measures. The cut off for significance was $p<.05$, two-tailed. The between-subjects factor was chronic haloperidol dose level and the within-subjects factor was acute nicotine dose.

\section{RESULTS}

\section{Simple and Complex Reaction Time}

There were no significant nicotine or haloperidol effects on simple reaction time (Figure 1). Even though there were no significant nicotine or haloperidol effects on simple reaction time, there were significant effects of both nicotine and haloperidol on spatial rotation time
(Figure 1). Haloperidol significantly increased reaction time $(\mathrm{F}(2,12)=5.23, p<.025)$ and nicotine significantly decreased it $(\mathrm{F}(3,36)=3.86, p<.025)$. There was also a significant nicotine $\times$ haloperidol interaction $(F(6,36)=$ $3.07, p<.025)$. Analyses of the simple main effects of nicotine in each of the three haloperidol treatment groups demonstrated differential effects of nicotine in these groups. No nicotine effect was seen in the low haloperidol dose group $(F(3,15)=0.77$, NS). In contrast, the medium haloperidol dose group showed a significant effect of nicotine $(\mathrm{F}(3,15)=3.92, p<.05)$ with a significant quadratic trend of the nicotine doses $(p<.025)$. The 7- $(p<.005)$ and $14-(p<.05)$ but not the $21-\mathrm{mg}$ nicotine patches significantly decreased reaction time relative to placebo. The high haloperidol group also showed a significant effect of nicotine $(\mathrm{F}(3,6)=6.52, p<.05)$ with a significant linear trend of nicotine doses $(p<$ $.01)$. The 14- $(p<.01)$ and 21- $(p<.025)$ but not the 7 -mg nicotine patches significantly decreased reaction time relative to placebo.

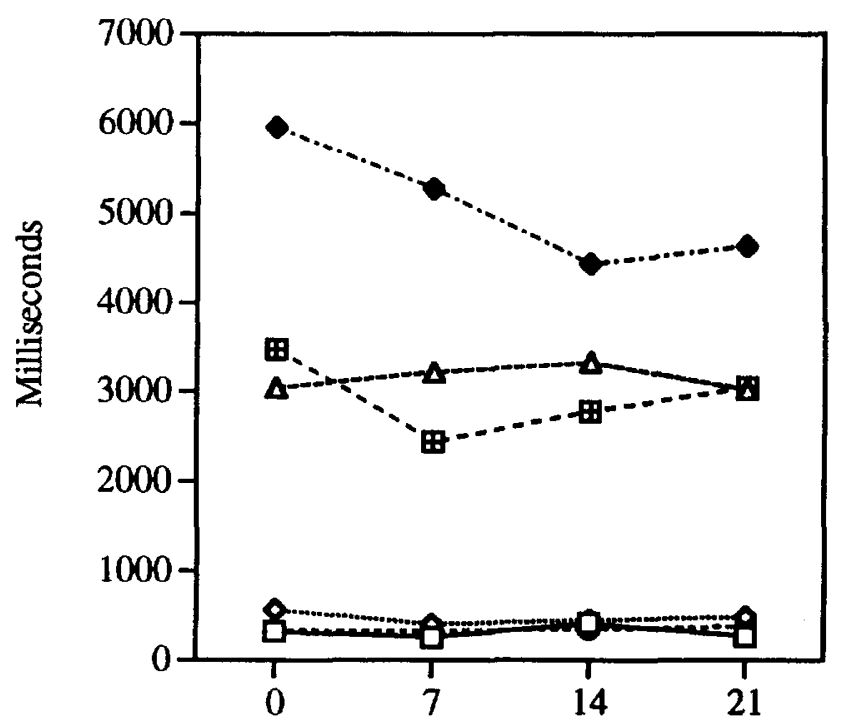

Nicotine Patch (mg)

Figure 1. Nicotine and haloperidol effects on the simple reaction time and complex (spatial rotation) reaction time (mean \pm SEM). For complex reaction time, main effect of nicotine $(p<.025)$, main effect of haloperidol $(p<.025)$, interaction of nicotine $\times$ haloperidol $(p<.025)$, For the medium haloperidol group, the nicotine effect was significant $(p<$ $.05)$ with a quadratic trend $(p<.025)$, nicotine $7 \mathrm{mg}(p<$ $.005)$ and $14 \mathrm{mg}(p<.05)$ patches reduced complex reaction time. For the high haloperidol group, the nicotine effect was significant $(p<.05)$ with a linear trend $(p<.01)$, nicotine 14 $\mathrm{mg}(p<.01)$ and $21 \mathrm{mg}(p<.025)$ patches reduced complex reaction time. Legend: spatial rotation reaction time for low haloperidol (open triangles), medium haloperidol (quadrants), and high haloperidol (solid diamonds); simple reaction time for low haloperidol (open squares), medium haloperidol (open diamond), and high haloperidol (open circles). 


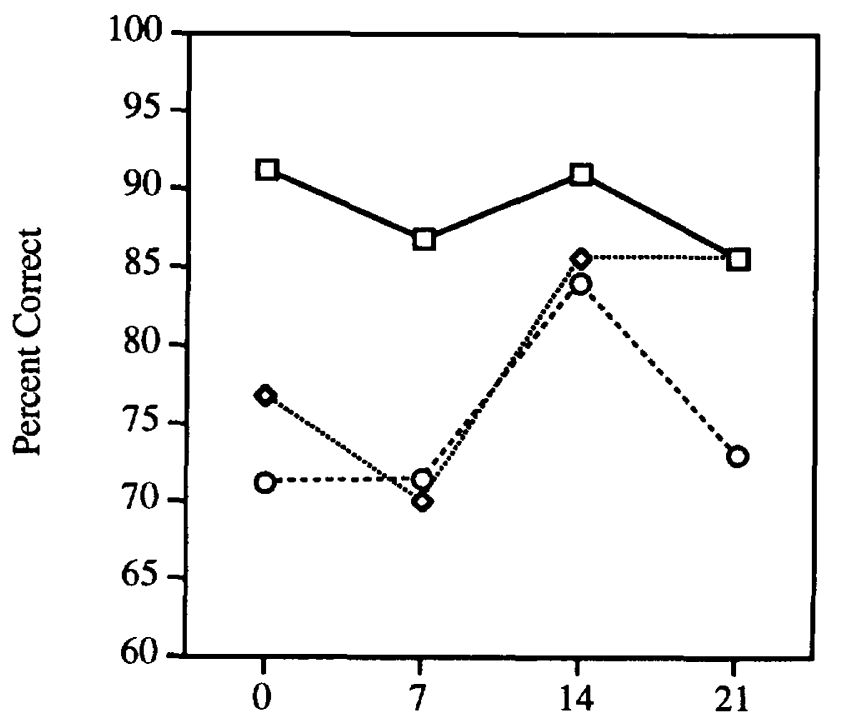

Nicotine (mg)

Figure 2. Nicotine and haloperidol interactions on delayed matching to sample percent correct (mean \pm SEM). In medium and high dose haloperidol groups, nicotine main effect $(p<.025)$ with a significant linear trend of improvement $(p<.05)$, nicotine 0 vs. $14 \mathrm{mg}(p<.05)$. Legend: low haloperidol (open squares), medium haloperidol (open diamonds), and high haloperidol (open circles).

\section{Delayed Matching to Sample}

There was no effect of nicotine in the low haloperidol group; however, when the medium and high haloperidol groups were considered there was a significant $(\mathrm{F}(3,24)=3.83, p<0.25)$ nicotine effect on this test (Figure 2). The medium and high haloperidol groups showed a significant linear improvement in performance with increasing nicotine doses $(p<.05)$. The 14$\mathrm{mg}$ nicotine patch dose significantly improved performance $(p<.05)$ and effectively reversed the haloperi- dol-induced deficit in the medium and high dose haloperidol groups.

\section{Sternberg Memory Test}

This test of verbal memory did not detect any haloperidol or nicotine effects.

\section{Conners Continuous Performance Test}

This test produced several measures of significant nicotine effects and one measure of haloperidol effect but no significant nicotine $\times$ haloperidol interactions. No significant main effects of either haloperidol or nicotine or significant interactions were seen in either errors of omission or errors of commission (Tables 1A and 1B). The principal effects were seen in terms of reaction time and its variability.

With hit reaction time, there was a significant main effect of nicotine $(\mathrm{F}(3,36)=3.01, p<.05)$. There was a significant quadratic trend of nicotine dose $(p<.05)$. As shown in Table $2 \mathrm{~A}$, reaction time decreased with the 7 - and $14-\mathrm{mg}$ patches and rose with the 21-mg patch. There were no significant effects of either haloperidol or nicotine on the standard error (SE) of reaction time (Table 2B). A nearly significant effect of nicotine was noted $(F(3,36)=$ $2.48, p<.08)$. Nearly significant decreased standard errors compared to the placebo nicotine patch were seen with the 7-mg $(p<.07)$ and $21-\mathrm{mg}$ patches $(p<.06)$.

Variability of SE of hit reaction time was assessed as an overall average and as it progressed over the blocks of trials in the session and as it progressed over the different interstimulus intervals. Average SE variability during a session (Table $3 \mathrm{~A}$ ) showed a nearly significant decreasing linear trend with increasing nicotine dose $(p<$ .06). The highest dose nicotine patch $(21 \mathrm{mg})$ caused a significant decrease in variability compared with placebo $(p<.05)$, with a decrease from $21.4 \pm 3.2$ with the placebo patch to $15.0 \pm 2.1$ with the $21-\mathrm{mg} /$ day patch.

Table 1. CPT Errors

\begin{tabular}{|c|c|c|c|c|}
\hline \multirow[b]{2}{*}{ Haloperidol Dose } & \multicolumn{4}{|c|}{ Nicotine Patch Strengths (mg/day) } \\
\hline & 0 & 7 & 14 & 21 \\
\hline \multicolumn{5}{|c|}{ A. CPT Errors of Omission } \\
\hline Low & $9.2 \pm 5.5$ & $4.8 \pm 3.1$ & $15.3 \pm 10.2$ & $6.0 \pm 2.7$ \\
\hline Medium & $18.8 \pm 9.6$ & $8.2 \pm 3.8$ & $6.7 \pm 2.1$ & $16.7 \pm 9.3$ \\
\hline High & $11.7 \pm 9.3$ & $19.3 \pm 11.9$ & $18.3 \pm 11.5$ & $17.0 \pm 13.5$ \\
\hline All & $13.5 \pm 4.6$ & $9.1 \pm 3.1$ & $12.5 \pm 4.6$ & $12.5 \pm 4.6$ \\
\hline \multicolumn{5}{|l|}{ B. Errors of Commission } \\
\hline Low & $17.3 \pm 3.0$ & $14.8 \pm 2.8$ & $14.3 \pm 3.1$ & $15.7 \pm 2.9$ \\
\hline Medium & $15.7 \pm 4.3$ & $20.7 \pm 4.2$ & $18.3 \pm 4.4$ & $14.8 \pm 2.2$ \\
\hline High & $17.0 \pm 5.5$ & $15.7 \pm 5.5$ & $17.7 \pm 5.2$ & $11.3 \pm 4.7$ \\
\hline All & $16.6 \pm 2.2$ & $17.3 \pm 2.2$ & $16.6 \pm 2.3$ & $14.5 \pm 1.6$ \\
\hline
\end{tabular}

Mean \pm SEM. 
Table 2. Hit Reaction Time

\begin{tabular}{|c|c|c|c|c|}
\hline \multirow[b]{2}{*}{ Haloperidol Dose } & \multicolumn{4}{|c|}{ Nicotine Patch Strengths (mg/day) } \\
\hline & $\mathbf{0}$ & 7 & 14 & 21 \\
\hline \multicolumn{5}{|c|}{ A. CPT Hit Reaction Time (msec) ${ }^{a}$} \\
\hline Low & $405 \pm 42$ & $394 \pm 39$ & $409 \pm 38$ & $384 \pm 29$ \\
\hline Medium & $365 \pm 40$ & $286 \pm 51$ & $355 \pm 38$ & $414 \pm 20$ \\
\hline High & $429 \pm 65$ & $416 \pm 96$ & $413 \pm 53$ & $463 \pm 50$ \\
\hline All & $394 \pm 25$ & $355 \pm 33$ & $388 \pm 24$ & $412 \pm 18$ \\
\hline \multicolumn{5}{|l|}{$\begin{array}{l}\text { B. Standard Error of CPT Hit } \\
\text { Reaction Time }{ }^{b}\end{array}$} \\
\hline Low & $10.9 \pm 1.7$ & $8.6 \pm 1.1$ & $10.2 \pm 1.6$ & $9.2 \pm 1.8$ \\
\hline Medium & $13.0 \pm 2.9$ & $11.4 \pm 2.2$ & $12.2 \pm 2.5$ & $10.7 \pm 2.3$ \\
\hline High & $14.9 \pm 4.7$ & $12.1 \pm 0.4$ & $16.2 \pm 4.7$ & $12.0 \pm 3.7$ \\
\hline All & $12.5 \pm 1.5$ & $10.4 \pm 1.0$ & $12.2 \pm 1.5$ & $10.4 \pm 1.3$ \\
\hline
\end{tabular}

Mean \pm SEM

${ }^{n}$ Quadratic trend of nicotine dose $(p<0.05)$.

${ }^{b}$ Nicotine 0 vs. $7 \mathrm{mg}(p<0.07)$; nicotine 0 vs. $21 \mathrm{mg}(p<0.06)$.

The progressive change in SE of hit reaction time was assessed over the course of blocks within a session (Table $3 \mathrm{~B}$ ) and over the different interstimulus intervals (Table 3C). With the change in SE of reaction time over the blocks of the session, there were significant main effects of both haloperidol $(\mathrm{F}(2,12)=4.46, p<.05)$ and nicotine $(\mathrm{F}(3,36)=2.90, p<.05)$. However, the nature of the drug effects was not clear. With haloperidol, there was no significant difference between the effects of low and medium doses. There was a nearly significant $(p<$ .06) increase in SE change over blocks with the high haloperidol group compared with the low dose group. With nicotine, the 7-mg patch caused a nearly significant $(p<.07)$ decrease in SE change over blocks compared with placebo, whereas there were no indications of differences between placebo and the 14- or $21-\mathrm{mg}$ nicotine patch conditions. With the change in SE of reaction time over the different interstimulus intervals, there was a significant main effect of nicotine $(F(3,36)=$ $5.77, p<.005)$, but not of haloperidol. There was a significant linear trend of nicotine decreasing SE $(p<.05)$. As shown in Table 3C, all three doses of nicotine produced lower mean scores, with the $7-\mathrm{mg}(p<.001)$ and $21-\mathrm{mg}(p<.005)$ patches scores significantly lower than the placebo.

\section{DISCUSSION}

These data show that nicotine was effective in attenuating and reversing the spatial memory and processing impairments caused by moderate and high doses of haloperidol. In addition, nicotine caused significant improvements in performance of schizophrenics regardless of haloperidol dose in terms of response consistency on the CPT task assessing attentiveness. Nicotine may be a useful adjunct to schizophrenia treatment regimens for attenuating the adverse side effects of antipsychotic drugs and for reducing cognitive deficits associated with schizophrenia.

The absence of significant effects with simple reaction time indicates that the effects on spatial rotation reaction time were not merely due to drug effects on motor speed. The drug effects seem to be more likely associated with changes in the speed of mental rotation of the sample stimulus to match comparison stimulus and changes in the time to choose the correct response.

The cognitive slowing, bradyphrenia, caused by haloperidol was evident in the spatial rotation task (choice reaction task). Nicotine reversed the slowing caused by the medium dose of haloperidol and significantly attenuated the more dramatic slowing caused by the high dose of haloperidol. Interestingly, this slowing did not seem to be related to just the reaction time. The simple reaction time test, which contained all of the sensory and motor components of the more complex task, did not detect any significant effects of either haloperidol or nicotine.

The delayed matching to sample task clearly demonstrated a memory impairment caused by haloperidol. Both the medium and high doses caused significant deficits relative to the low dose when no nicotine was administered. The deficits were still apparent with the $7-\mathrm{mg} /$ day dose of nicotine. The 14-mg/day dose of nicotine was effective in attenuating the haloperidol-induced deficits in both the medium and high dose haloperidol groups. The $21-\mathrm{mg} /$ day dose of nicotine was effective in eliminating the deficit in the medium haloperidol group. With the high dose haloperidol group the 21$\mathrm{mg} /$ day dose had less efficacy than the 14-mg/day dose, providing evidence for an inverted U-shaped nicotine dose-effect function for this group. This is often seen with cognitive enhancing drugs.

In contrast to the delayed matching to sample test, there were no effects of haloperidol and nicotine on the 
Table 3. CPT Hit Reaction Times

\begin{tabular}{|c|c|c|c|c|}
\hline \multirow[b]{2}{*}{ Haloperidol Dose } & \multicolumn{4}{|c|}{ Nicotine Patch Strengths (mg/day) } \\
\hline & $\mathbf{0}$ & 7 & 14 & 21 \\
\hline \multicolumn{5}{|c|}{ A. CPT Hit Reaction Time SE Variability ${ }^{a}$} \\
\hline Low & $18.2 \pm 4.6$ & $11.4 \pm 1.8$ & $15.0 \pm 2.4$ & $13.8 \pm 3.8$ \\
\hline Medium & $23.9 \pm 6.2$ & $21.3 \pm 5.1$ & $21.8 \pm 7.0$ & $15.5 \pm 3.6$ \\
\hline High & $22.8 \pm 5.7$ & $21.0 \pm 4.6$ & $20.4 \pm 6.8$ & $16.4 \pm 5.0$ \\
\hline All & $21.4 \pm 3.2$ & $17.3 \pm 2.5$ & $18.8 \pm 3.1$ & $15.0 \pm 2.1^{*}$ \\
\hline \multicolumn{5}{|c|}{$\begin{array}{l}\text { B. CPT Hit Reaction Time SE Change over } \\
\text { Session Blocks }{ }^{b}\end{array}$} \\
\hline Low & $0.08 \pm 0.03$ & $0.00 \pm 0.02$ & $0.05 \pm 0.03$ & $0.04 \pm 0.03$ \\
\hline Medium & $-0.06 \pm 0.06$ & $-0.04 \pm 0.04$ & $0.06 \pm 0.05$ & $-0.03 \pm 0.03$ \\
\hline High & $0.14 \pm 0.04$ & $0.01 \pm 0.02$ & $0.13 \pm 0.02$ & $0.05 \pm 0.04$ \\
\hline All & $0.04 \pm 0.04$ & $-0.01 \pm 0.02$ & $0.07 \pm 0.02$ & $0.02 \pm 0.02$ \\
\hline \multicolumn{5}{|c|}{$\begin{array}{l}\text { C. CPT Hit Reaction Time SE Change over } \\
\text { Interstimulus Intervals }{ }^{c}\end{array}$} \\
\hline Low & $0.14 \pm 0.06$ & $0.02 \pm 0.04$ & $0.03 \pm 0.02$ & $0.04 \pm 0.05$ \\
\hline Medium & $0.06 \pm 0.05$ & $-0.03 \pm 0.07$ & $0.03 \pm 0.05$ & $-0.03 \pm 0.05$ \\
\hline High & $0.11 \pm 0.04$ & $-0.13 \pm 0.08$ & $0.11 \pm 0.01$ & $-0.08 \pm 0.01$ \\
\hline All & $0.10 \pm 0.03$ & $-0.03 \pm 0.04^{* * *}$ & $0.05 \pm 0.02$ & $-0.01 \pm 0.03^{* *}$ \\
\hline
\end{tabular}

Sternberg memory test. This may have been related to the different nature of the memory task. The Sternberg test assesses verbal nonspatial memory, whereas the delayed matching to sample assesses spatial memory. Spatial and nonspatial memory have been shown in a variety of studies to have different critical neural substrates (Levin et al. 1992). Both the delayed matching to sample and complex reaction time tests had important spatial components. This spatial aspect may have been critical for the haloperidol and nicotine effects seen in the current study. In animal models, disruption of dopaminergic transmission of the frontal cortex or the limbic system has been found to cause impaired performance on spatial tasks (Le Moal and Simon 1991).

There are mixed results concerning neuroleptic effects on cognitive function. Cleghorn et al. (1990) found that neuroleptics impaired nonverbal memory, whereas Medalia et al. (1988) did not. Verdoux et al. (1995) assessed the cognitive performance of schizophrenics first off and then on neuroleptic medication (haloperidol, fluphenazine, or chlorpromazine). They found that the subjects significantly improved on the digit-symbol substitution test from the WAIS-R battery and the Stroop color naming test on the retest when they were on neuroleptic medication. Unfortunately, in this study there was no control for the confound of test experience and drug administration. This seems to be a real concern, because they found more significant improvements in these tests with repeated experience in normal unmedicated subjects. The current results add to this literature with the finding of neuroleptic-induced spatial but not verbal memory impairment.

Benztropine (Cogentin) was administered in doses of 1 or $2 \mathrm{mg} /$ day to patients who were experiencing Parkinsonian-like effects of haloperidol. Benztropine has muscarinic cholinergic antagonist actions and also has dopaminergic agonist-like effects (Modell et al. 1989). Either of these actions can have effects on the tests administered. However, in a separate set of analyses using benztropine (0, 1, and $2 \mathrm{mg}$ doses) as a factor did not uncover any significant effects of this drug on any of the measures taken.

CPT tests have previously been shown to be sensitive to schizophrenia-associated deficits in attentiveness (Benedict et al. 1994; Pandurangi et al. 1994). In the current study, the CPT test showed that nicotine reduced the variability in response speed across the different interstimulus intervals. This effect was not differently expressed in the three different haloperidol dose groups. This reduction in variability of response is similar to the effect of nicotine we have previously seen in adults with attention deficit/hyperactivity disorder (Levin et al. 1996).

These results suggest that schizophrenics may smoke cigarettes at least partially to attenuate the adverse side effects of antipsychotic drugs. Decina et al. (1990) found that schizophrenics who smoked had significantly lower rates of neuroleptic-induced Parkinsonism. Nicotine is quite effective in stimulating the release of DA (Wonna- 
cott et al. 1989), an effect that may help it to reverse the adverse side effects of haloperidol. In particular, dopamine $\mathrm{D}_{2}$ blockade may be important. We have found that haloperidol, a potent $\mathrm{D}_{2}$ antagonist causes dose-related increases in smoking (McEvoy et al. 1995a), whereas clozapine, an atypical neuroleptic with little $D_{2}$ antagonistic effect actually caused a dose-related decrease in smoking (McEvoy et al. 1995b). Because nicotinic receptors are quite easily desensitized, it is not clear at this time whether the therapeutic effects seen in the current study resulted from receptor stimulation or receptor desensitization caused by nicotine administered via the skin patch.

It is important to acknowledge that all of the subjects in the current study were smokers who had been deprived of cigarettes. As such they would have been in a state of nicotine withdrawal at the time of testing. The effects of haloperidol may have been to exacerbate the adverse effects of nicotine withdrawal.

This study sheds light on both basic and applied problems. On the basic side, it appears that nicotine and DA systems have important interactions with regard to cognitive processes. This interaction especially seems to be important with regard to spatial processing. On the applied side, these results provide important information concerning the very high smoking rates in schizophrenia. Attempts to encourage schizophrenics to quit smoking should probably include nicotine replacement. It is an intriguing possibility that nicotine administered via the skin patch or other nicotinic agonists may be useful as adjuncts in the treatment of schizophrenia.

\section{ACKNOWLEDGMENT}

This research was supported by a grant from the National Alliance for Research on Schizophrenia and Depression and NIDA Grant DA-08434.

\section{REFERENCES}

Adler LE, Hoffer LD, Wiser A, Freedman R (1993): Normalization of auditory physiology by cigarette smoking in schizophrenic patients. Am J Psychiatry 150:1856-1861

Andersson K, Fuxe K, Agnati LF (1981a): Effects of a single injection of nicotine on the ascending dopaminergic pathways in rats. Evidence for increased dopamine turnover in the mesostriatal and mesolimbic dopamine neurons. Acta Physiol Scand 112:345-347

Andersson K, Fuxe K, Agnati LF, Eneroth P (1981b): Effects of acute central and peripheral administration of nicotine on ascending dopamine pathways in male rat brain. Evidence for nicotine induced increases of dopamine turnover in various telencephalic dopamine nerve terminal systems. Med Biol 59:170-176

Benedict RHB, Harris AE, Markow T, McCormick JA, Nuechterlein KH, Asarnow RF (1994): Effects of atten- tion training on information processing in schizophrenia. Schizophr Bull 20:537-546

Clarke PBS (1990): Dopaminergic mechanisms in the locomotor stimulant effects of nicotine. Biochem Pharmacol 40:1427-1432

Cleghorn JM, Kaplan RD, Szechtman B, Szechtman H, Brown GM (1990): Neuroleptic drug effects on cognitive function in schizophrenia. Schizophr Res 3:211-219

Conners CK (1994): The Continuous Performance Test (CPT): Use as a diagnostic tool and measure of treatment outcome. Annual Convention of the American Psychological Association, Los Angeles, CA, Aug 12-16, 1994

Conners CK (1995): The Continuous Performance Test. Computer Program, Toronto, Multi-Health Systems

Dawe S, Gerada C, Russell MAH, Gray JA (1995): Nicotine intake in smokers increases following a single dose of haloperidol. Psychopharmacology 117:110-115

Decina P, Caracci G, Sandik R, Berman W, Mukherjee S, Scapicchio P (1990): Cigarette smoking and neurolepticinduced Parkinsonism. Biol Psychiatry 28:502-508

Fiore MC (1992): Trends in smoking in the United States. The epidemiology of tobacco use. Med Clin North Am 76:289303

Gras-Vincendon A, Danion JM, Grange D, Bilik M, WillardSchroeder D, Sichel JP, Singer L (1994): Explicit memory, repetition priming and cognitive skill learning in schizophrenia. Schizophr Res 13:117-126

Grenhoff J, Aston-Jones G, Svensson TH (1986): Nicotinic effects on the firing pattern of midbrain dopamine neurons. Acta Physiol Scand 128:151-158

Hughes JR, Hatsukami DK, Mitchell JE, Dahlgren LA (1986): Prevalence of smoking among psychiatric outpatients. Am J Psychiatry 143:993-997

Jarvik ME, Caskey NH, Wirshing WC (1996): Dopaminergic drugs modulate cigarette smoking. Addiction 91:141

Le Moal M, Simon H (1991): Mesocortical dopaminergic network: Functional and regulatory roles. Physiol Rev 71:155-234

Levin ED (1992): Nicotinic systems and cognitive function. Psychopharmacology 108:417-431

Levin ED, Schantz SL, Bowman RE (1992): Use of the lesion model for examining toxicant effects on cognitive behavior. Neurotoxicol Teratol 14:131-141

Levin ED, Conners CK, Sparrow E, Hinton S, Meck W, Rose JE, Ernhardt D, March J (1996): Nicotine effects on attention-deficit/hyperactivity disorder. Psychopharmacology 123:55-63

Lichtensteiger W, Hefti F, Felix D, Huwyler T, Melamed E, Schlumpf M (1982): Stimulation of nigrostriatal dopamine neurons by nicotine. Neuropharmacology 21:963-968

Lohr JB, Flynn K (1992): Smoking and schizophrenia. Schizophr Res 8:93-102

McEvoy J, Freudenreich O, Levin E, Rose J (1995a): Haloperidol increases smoking in patients with schizophrenia. Psychopharmacology 119:124-126

McEvoy J, Freudenreich O, McGee M, VanderZwaag C, Levin E, Rose J (1995b): Clozapine decreases smoking in patients with chronic schizophrenia. Biol Psychiatry 37:550-552

Medalia A, Gold J, Merriam A (1988): The effects of neuroleptics on neuropsychological test results of schizophrenics. Arch Clin Neuropsychol 3:249-271 
Modell J, Tandon R, Beresford T (1989): Dopaminergic activity of the antiparkinsonian agents. J Clin Psychopharmacol 9:347-351

O'Farrell TJ, Connors GJ, Upper D (1983): Addictive behaviors among hospitalized schizophrenic patients. Addict Behav 8:329-333

Pandurangi AK, Sax KW, Pelonero AL, Goldberg SC (1994); Sustained attention and positive formal thought disorder in schizophrenia. Schizophr Res 13:109-116

Reeves D, Bleiberg J, Spector J (1993a): Validation of the ANAM battery in multi-center head injury rehabilitation studies. Arch Clin Neuropsychol 8:356
Reeves D, Kane R, Winter K, Raynsford K, Pancella T (1993b): Automated Neuropsychological Assessment Metrics (ANAM): Test Administrator's Guide Version 1.0. St. Louis, Missouri, Institute of Mental Health

Verdoux H, Magnin E, Bourgeois M (1995): Neuroleptic effects on neuropsychological test performance in schizophrenia. Schizophr Res 14:133-139

Wonnacott S, Irons I, Rapier C, Thorne B, Lunt GG (1989): Presynaptic modulation of transmitter release by nicotinic receptors. In Nordberg A, Fuxe K, Holmstedt B, Sundwall A (eds), Progress in Brain Research. New York, Elsevier Science Publishers B.V., pp 157-163 